\title{
Trade-offs in Robustness to Perturbations of Bacterial Population Controllers*
}

\author{
Cameron $\mathrm{McBride}^{1 \dagger}$ and Domitilla Del Vecchio ${ }^{1}$
}

\begin{abstract}
Synthetic biology applications have the potential to have lasting impact; however, there is considerable difficulty in scaling up engineered genetic circuits. One of the current hurdles is resource sharing, where different circuit components become implicitly coupled through the host cell's pool of resources, which may destroy circuit function. One potential solution around this problem is to distribute genetic circuit components across multiple cell strains and control the cell population size using a population controller. In these situations, perturbations in the availability of cellular resources, such as due to resource sharing, will affect the performance of the population controller. In this work, we model a genetic population controller implemented by a genetic circuit while considering perturbations in the availability of cellular resources. We analyze how these intracellular perturbations and extracellular disturbances to cell growth affect cell population size. We find that it is not possible to tune the population controller's gain such that the population density is robust to both extracellular disturbances and perturbations to the pool of available resources.
\end{abstract}

\section{INTRODUCTION}

Many exciting applications exist for engineered biological systems in fields ranging from medicine to the environment [1]-[4]. In order to create sufficiently sophisticated engineered systems that can be applied in practice, substantial research has gone into techniques to scale up the size and complexity of genetic circuits. One major hurdle to scaling up a circuit's size is resource sharing, where different circuit components become undesirably coupled through the host cell's pool of resources [5], [6] and affects cell growth [7]-[9]. Solutions to the resource sharing problem have recently appeared. Some solutions make genetic modules robust to resource fluctuations with feedback control [10], [11], some engineer separate pool of resources for the engineered circuits [12], and others distribute the circuit across multiple cell strains [13], [14].

Some highly complex circuits have been created by distributing genetic circuits across different cell strains [14], [16]-[22]. Many of these systems utilize microfluidic platforms or spacial separation to isolate bacterial

\footnotetext{
*This work was supported the National Science Foundation CCF Award \# 1521925

${ }^{1}$ Massachusetts Institute of Technology, Department of Mechanical Engineering, 77 Massachusetts Ave, Cambridge, MA 02139, USA cmcbride@mit. edu, and ddv@mit. edu

$\dagger$ Corresponding Author
}

strains from one another. However, if multiple cell strains are not spatially separated, population control is required to maintain a desired cell population size [20]. In future applications where genetic circuits are distributed across cells and each cell also contains a population controller, the population controller's robustness to perturbations in cellular resources, for instance, those applied by the other genetic circuits, will influence the overall system's function.

In particular, in a setup where the population controller operates concurrently with other genetic circuits, such as with a sensor or a more sophisticated circuit that releases pharmaceutical proteins, perturbations to the pool of resources due to these circuits affect the population controller as a disturbance. Meanwhile, changes in the availability of nutrients in the environment, for example, due to the presence of other cell strains competing for nutrients, affect the dynamics of the cell population size that the controller is attempting to regulate as shown in Figure 1. Here, we specifically focus on the potential trade-off between robustness to extracellular perturbations due to the presence of other cells competing for nutrients ( $W$ in Figure 1 ), and to intracellular perturbations to the pool of cell's resources due to the expression of other genetic circuits ( $\mathrm{X}$ in Figure 1). In related work, [15] considers a decrease in growth rate due to the expression of enzymes and finds a condition on the genetic circuit parameters where dividing the production of a metabolite across multiple cell strains results in higher yield; however, perturbations in the availability of cellular resources and environmental disturbances are not considered.

This paper is organized as follows. In Section II, we create a mechanistic model for cell growth and the population controller. In Section III, we present our main results by analyzing this model for its sensitivity to disturbances in the environment and to fluctuations in available cellular resources. We conclude in Section IV.

\section{Model of a Population Control System}

We consider the cellular population control circuit of [26], shown in Figure 1. This controller has been shown to maintain a constant number of cells in a bacterial population significantly below the carrying capacity of the environment under controlled laboratory conditions. 
This population control architecture has been used in most of the applications of genetic circuits distributed across multiple cells [20]. We create a model to describe the population dynamics, then we will model the dynamics of the population controller. We will use this system model to analyze how the number of cells in the population changes with extracellular disturbances, for example, due to competing cell strains, and with intracellular disturbances, for example, due to the expression of additional synthetic genes within the cell [5].

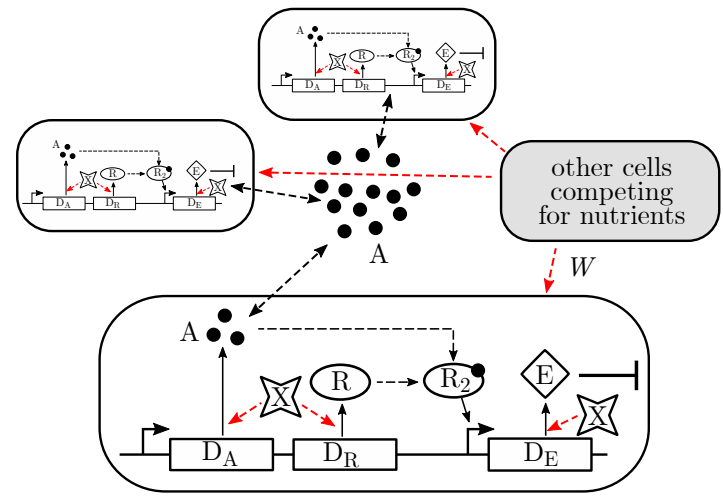

Fig. 1. Population control genetic circuit. The metabolite $\mathrm{A}$ is produced from DNA $\mathrm{D}_{\mathrm{A}}$. The metabolite A diffuses through the cell membrane into the environment. A also diffuses into the cell and binds to $\mathrm{R}$ to become $\mathrm{R}_{2}$. The $\mathrm{R}_{2}$ complex then activates the production of the killing gene $\mathrm{E}$, which increases the cell death rate. Each protein production step requires cellular resources $\mathrm{X}$ to function. Other cells in the environment compete for nutrients with the strain of interest, resulting in the disturbance $W$. We consider $W$ and fluctuations in X as disturbance inputs.

Considering the situation shown in Figure 1, let the number of cells in the bacterial population of interest be $N$. We model the population size with the logistic growth model, which is standard for bacteria [26], [27]. Then, the dynamics of the cell population size are given as

$$
\dot{N}=\mu N\left(1-\frac{N+W}{K}\right)-\left(\gamma_{N}+G E\right) N,
$$

where $\mu$ is the maximum growth rate of the cell line, $K$ is the carrying capacity of the environment, $\gamma_{N}$ is the basal rate of cell death, $E$ is the concentration of the killing protein $\mathrm{E}$, and $G$ is the effect of the concentration of the killing protein on the population death rate. Additionally, $W$ represents an environmental disturbance such as the presence of other cell strains, which reduces the available nutrients to our cell strain of interest.

Next, we model the biochemical species that control the cell population size $N$ through the production of the killing protein $\mathrm{E}$ as shown in Figure 1. In this system, each cell produces the small molecule $\mathrm{A}$ at a constant rate. Here, we model the generation of this molecule as a simple gene expression process. Then, molecule A diffuses through the cellular membrane into the surrounding media. We assume that the diffusion of A occurs quickly and the media is well mixed so the concentration of $\mathrm{A}$ is uniform throughout (both within and outside the cell). As the concentration of A becomes large due the presence of a large number of cells, A diffuses into cells and binds to $\mathrm{R}$, which is expressed at a constant rate. The active complex of $\mathrm{R}$ with $\mathrm{A}$ is represented by $R_{2}$. The $R_{2}$ complex then activates the production of the killing protein $\mathrm{E}$, which kills some of the cells, decreasing the number of cells in the population [26]. This population controller structure is based off a common quorum sensing motif found in multiple natural bacteria [24], [25].

In the model, we include resources $\mathrm{X}$ required for gene expression as indicated in Figure 1. Specifically, we lump together the processes of transcription and translation [28], so that we can view $\mathrm{X}$ as a lumped resource required for protein production. Additionally, we consider the free resources as an input for simplicity. More realistic models may consider the free resources as a state that depends on all proteins being produced in the cell [28] with the activation or repression of other circuits appearing as the new disturbance, imparting a change in X. We ignore any interaction between resource fluctuations and cell growth and assume that protein degradation and dilution remain constant and are independent of population size. Under these assumptions, the chemical reactions describing the biochemical controller are given as

$$
\begin{aligned}
& \mathrm{D}_{\mathrm{A}}+\mathrm{X} \underset{d_{A}}{\stackrel{a_{A}}{\rightleftharpoons}} \mathrm{C}_{\mathrm{A}} \stackrel{k_{A}}{\longrightarrow} \mathrm{A}+\mathrm{D}_{\mathrm{A}}+\mathrm{X}, \quad(\mathrm{N} \text { times }) \\
& \mathrm{D}_{\mathrm{R}}+\mathrm{X} \underset{d_{R}}{\stackrel{a_{R}}{\rightleftharpoons}} \mathrm{C}_{\mathrm{R}} \stackrel{k_{R}}{\longrightarrow} \mathrm{R}+\mathrm{D}_{\mathrm{R}}+\mathrm{X} \\
& \mathrm{R}+\mathrm{A} \underset{d_{R_{2}}}{\stackrel{a_{R_{2}}}{\rightleftharpoons}} \mathrm{R}_{2} \\
& \mathrm{D}_{\mathrm{E}}+\mathrm{X}+\mathrm{R}_{2} \underset{d_{E}}{\stackrel{a_{E}}{\rightleftharpoons}} \mathrm{C}_{\mathrm{E}} \stackrel{k_{E}}{\longrightarrow} \mathrm{E}+\mathrm{D}_{\mathrm{E}}+\mathrm{X}+\mathrm{R}_{2} \\
& \mathrm{C}_{\mathrm{A}} \stackrel{\gamma}{\rightarrow} \mathrm{D}_{\mathrm{A}}+\mathrm{X}, \quad \mathrm{C}_{\mathrm{R}} \stackrel{\gamma}{\rightarrow} \mathrm{D}_{\mathrm{R}}+\mathrm{X} \\
& \mathrm{C}_{\mathrm{E}} \stackrel{\gamma}{\rightarrow} \mathrm{D}_{\mathrm{E}}+\mathrm{X}+\mathrm{R}_{2} \\
& \mathrm{~A} \stackrel{\gamma_{A}}{\rightarrow} \emptyset, \quad \mathrm{R} \stackrel{\gamma}{\rightarrow} \emptyset, \quad \mathrm{R}_{2} \stackrel{\gamma}{\rightarrow} \emptyset, \quad \mathrm{E} \stackrel{\gamma}{\rightarrow} \emptyset,
\end{aligned}
$$

where $D_{A}, D_{R}$, and $D_{E}$ are the DNA molecules that produce $A, R$ and $E$, respectively. Since A diffuses through the cellular membrane and the concentration of $\mathrm{A}$ is uniform throughout the media, the production rate of A is proportional to the cell population size $N$ since each cell produces $\mathrm{A}$ and $N$ is dimensionless. Then, using the law of mass-action [29], the dynamics of the concentration of the biochemical controller species are 
given as

$$
\begin{aligned}
\dot{A} & =k_{A} C_{A} N-a_{R_{2}} A R+d_{R_{2}} R_{2}-\gamma_{A} A \\
\dot{R} & =k_{R} C_{R}-a_{R_{2}} A R+d_{R_{2}} R_{2}-\gamma R \\
\dot{E} & =k_{E} C_{E}-\gamma E \\
\dot{R}_{2}= & a_{R_{2}} A R-d_{R_{2}} R_{2}-a_{E} D_{E} R_{2} X \\
& \quad+\left(d_{E}+k_{E}+\gamma\right) C_{E}-\gamma R_{2} \\
\dot{C}_{A}= & a_{A} D_{A} X-\left(d_{A}+k_{A}+\gamma\right) C_{A} \\
\dot{C}_{R}= & a_{R} D_{R} X-\left(d_{R}+k_{R}+\gamma\right) C_{R} \\
\dot{C}_{E}= & a_{E} D_{E} R_{2} X-\left(d_{E}+k_{E}+\gamma\right) C_{E},
\end{aligned}
$$

with the conservation laws for the DNA

$$
\begin{aligned}
& D_{A_{t o t}}=D_{A}+C_{A} \\
& D_{R_{t o t}}=D_{R}+C_{R} \\
& D_{E_{t o t}}=D_{E}+C_{E} .
\end{aligned}
$$

Next, we utilize timescale separation to reduce the dimension of (2), since we are interested in the dynamics of this system on the slow timescale. Since binding and unbinding reactions occur much faster than protein production and degradation [28], i.e. $d_{R_{2}}, d_{A}, d_{R}, d_{E} \gg$ $\gamma$, we define a small parameter $\varepsilon \triangleq \frac{\gamma}{d_{R_{2}}} \ll 1$ and the parameters $K_{A}=\frac{d_{A}}{a_{A}}, K_{R}=\frac{d_{R}}{a_{R}}, K_{e}=\frac{d_{E}}{a_{E}}, K_{R_{2}}=$ $\frac{d_{R_{2}}}{a_{R_{2}}}, \theta_{R_{2} A}=\frac{d_{A}}{d_{R_{2}}}, \theta_{R_{2} R}=\frac{d_{R}}{d_{R_{2}}}$, and $\theta_{R_{2} E}=\frac{d_{E}}{d_{R_{2}}}$. Then $R_{2}, C_{A}, C_{R}$, and $C_{E}$ are fast variables, while $A$ and $R$ are mixed variables, and $E$ is a slow variable. We apply a change of coordinates and define the new states $z_{A}=A+R_{2}+C_{E}$ and $z_{R}=R+R_{2}+C_{E}$, which are slow variables. Then, the system can be written in standard singular perturbation form [30] as

$$
\begin{aligned}
& \dot{z}_{A}=k_{A} C_{A} N-\gamma_{A}\left(z_{A}-R_{2}-C_{E}\right)-\gamma R_{2} \\
& \dot{z}_{R}=k_{R} C_{R}-\gamma\left(z_{R}-C_{E}\right) \\
& \dot{e}=k_{E} C_{E}-\gamma E \\
& \varepsilon \dot{R}_{2}=\gamma\left(\frac{A R}{K_{R_{2}}}-R_{2}(1+\varepsilon)\right. \\
&\left.-\theta_{R_{2} E}\left[\frac{R_{2} D_{E} X}{K_{E}}+C_{E}\left(1+\varepsilon \frac{k_{E}+\gamma}{\gamma \theta_{R_{2 E}}}\right)\right]\right) \\
& \varepsilon \dot{C}_{A}=\gamma \theta_{R_{2} A}\left(\frac{D_{A} X}{K_{A}}-C_{A}-\varepsilon \frac{k_{A}+\gamma}{\gamma \theta_{R_{2}}} C_{A}\right) \\
& \varepsilon \dot{C}_{R}=\gamma \theta_{R_{2} R}\left(\frac{D_{R} X}{K_{R}}-C_{R}-\varepsilon \frac{k_{R}+\gamma}{\gamma \theta_{R_{2} R}} C_{R}\right) \\
& \varepsilon \dot{C}_{E}=\gamma \theta_{R_{2} E}\left(\frac{D_{E} X}{K_{E}}-C_{E}-\varepsilon \frac{k_{E}+\gamma}{\gamma \theta_{R_{2} E}} C_{E}\right)
\end{aligned}
$$

with $z_{A}, z_{R}$, and $E$ as slow variables. Next, we let $\varepsilon \rightarrow$
0 . Then, the slow manifold is given as

$$
\begin{aligned}
C_{A} & =\frac{D_{A} X}{K_{A}}=\frac{D_{A_{t o t}} \frac{X}{K_{A}}}{1+\frac{X}{K_{A}}} \\
C_{R} & =\frac{D_{R} X}{K_{R}}=\frac{D_{R_{t o t} \frac{X}{K_{R}}}}{1+\frac{X}{K_{R}}} \\
C_{E} & =\frac{R_{2} D_{E} X}{K_{E}}=\frac{D_{E_{t o t}} \frac{R_{2} X}{K_{E}}}{1+\frac{R_{2} X}{K_{E}}} \triangleq g_{C_{E}}\left(R_{2}\right) \\
R_{2} & =\frac{A R}{K_{R_{2}}} \triangleq g_{R_{2}}(A, R) .
\end{aligned}
$$

The slow manifold is exponentially stable, which is verified by checking that the eigenvalues of the Jacobian of the fast states evaluated on the slow manifold (4d)-(4g) have uniformly negative real parts [30]. The Jacobian computed on the fast manifold is given as

$$
\gamma\left[\begin{array}{cccc}
a_{11} & 0 & 0 & a_{14} \\
0 & a_{22} & 0 & 0 \\
0 & 0 & a_{33} & 0 \\
a_{41} & 0 & 0 & a_{44}
\end{array}\right]
$$

where $a_{11}=-1-\frac{(A+R)}{K_{R_{2}}}-\frac{\theta_{R_{2} e} X}{K_{e}} D_{e}<0, a_{22}=$ $-\theta_{R_{2} A}\left(1+\frac{X}{K_{A}}\right)<0, a_{33}=-\theta_{R_{2} R}\left(1+\frac{X}{K_{R}}\right)<0$, $a_{44}=-\theta_{R_{2} E}\left(1+\frac{R_{2} X}{K_{E}}\right)<0, a_{14}=\frac{-(A+R)}{K_{R_{2}}}+$ $\theta_{R_{2} E}\left(1+\frac{R_{2} X}{K_{E}}\right)$, and $a_{41}=\gamma \theta_{R_{2} E} \frac{X}{K_{E}} D_{E}$. It can be shown that the real part of all eigenvalues of (6) are uniformly negative if $a_{22}, a_{33}<0$, which are always satisfied since concentrations are always nonnegative, and if $a_{11} a_{44}>a_{14} a_{41}$. The latter inequality reduces to

$$
1+\frac{X R_{2}}{K_{E}}+\frac{(A+R)}{K_{R_{2}}}\left(1+\frac{X\left(R_{2}+D_{E}\right)}{K_{E}}\right)>0
$$

which is also always satisfied since concentrations are nonnegative. Thus, the slow manifold is exponentially stable. Next, substituting (5) into the dynamics of the slow variables with the conservation laws (3), and defining $\alpha_{A}=k_{A} D_{A_{t o t}}, \alpha_{R}=k_{R} D_{R_{t o t}}$, and $\alpha_{E}=$ $k_{E} D_{E_{\text {tot }}}$, we have the reduced dynamics of the slow variables given by

$$
\begin{aligned}
\dot{z}_{A} & =N \frac{\alpha_{A} X}{K_{A}+X}-A\left(\gamma_{A}+\gamma \frac{R}{K_{R_{2}}}\right) \\
\dot{z}_{R} & =\frac{\alpha_{R} X}{K_{R}+X}-\gamma R\left(1+\frac{A}{K_{R_{2}}}\right) \\
\dot{E} & =\frac{\alpha_{e} A R X}{K_{R_{2}} K_{e}+A R X}-\gamma E .
\end{aligned}
$$

We now change variables back to the original variables $A$ and $R$ where the relation of the time derivatives 
between $z_{A}$ and $z_{R}$ to $A$ and $R$ are given as

$$
\begin{gathered}
\dot{z}_{A}=\dot{A}+\left(\dot{A} \frac{d g_{R_{2}}}{d A}+\dot{R} \frac{d g_{R_{2}}}{d R}\right)\left(1+\frac{d g_{C_{E}}}{d R_{2}}\right) \\
\dot{z}_{R}=\dot{R}+\left(\dot{A} \frac{d g_{R_{2}}}{d A}+\dot{R} \frac{d g_{R_{2}}}{d R}\right)\left(1+\frac{d g_{C_{E}}}{d R_{2}}\right) .
\end{gathered}
$$

Solving (8) for $\dot{A}$ and $\dot{R}$, we have

$$
\begin{aligned}
& \dot{R}=\frac{\dot{z}_{R}\left(1+\frac{d g_{R_{2}}}{d A}\left(1+\frac{d g_{C_{e}}}{d R_{2}}\right)\right)-\dot{z}_{A} \frac{d g_{R_{2}}}{d A}\left(1+\frac{d g_{C_{E}}}{d R_{2}}\right)}{1+\left(1+\frac{d g_{C_{E}}}{d R_{2}}\right)\left(\frac{d g_{R_{2}}}{d A}+\frac{d g_{R_{2}}}{d R}\right)} \\
& \dot{A}=\frac{\dot{z}_{A}\left(1+\frac{d g_{R_{2}}}{d R}\left(1+\frac{d g_{C_{e}}}{d R_{2}}\right)\right)-\dot{z}_{R} \frac{d g_{R_{2}}}{d R}\left(1+\frac{d g_{C_{E}}}{d R_{2}}\right)}{1+\left(1+\frac{d g_{C_{E}}}{d R_{2}}\right)\left(\frac{d g_{R_{2}}}{d A}+\frac{d g_{R_{2}}}{d R}\right)} .
\end{aligned}
$$

Next, we substitute (7) into (9). We assume that $\frac{A+R}{K_{R_{2}}} \ll$ $1, \frac{R}{K_{R_{2}}} \frac{\dot{z}_{A}}{\dot{z}_{R}}\left(1+\frac{d g_{C_{e}}}{d R_{2}}\right) \ll 1$, and $\frac{A}{K_{R_{2}}} \dot{\dot{z}}_{R} \dot{z}_{A}\left(1+\frac{d g_{C_{e}}}{d R_{2}}\right) \ll$ 1 , which can be guaranteed if binding between $\mathrm{A}$ and $\mathrm{R}$ is sufficiently weak. Then, system (9) takes the approximate form

$$
\begin{aligned}
\dot{N} & =\mu N\left(1-\frac{N+W}{K}\right)-\left(\gamma_{N}+G E\right) N \\
\dot{A} & =N \frac{\alpha_{A} X}{K_{A}+X}-A\left(\gamma_{A}+\gamma \frac{R}{K_{R_{2}}}\right) \\
\dot{R} & =\frac{\alpha_{R} X}{K_{R}+X}-\gamma R\left(1+\frac{A}{K_{R_{2}}}\right) \\
\dot{E} & =\frac{\alpha_{E} A R X}{K_{R_{2}} K_{E}+A R X}-\gamma E .
\end{aligned}
$$

In this model, $N$ is the output variable of interest while $W$ and $X$ are disturbances, which affect the population dynamics and the biomolecular feedback controller, respectively, as shown in Figure 2. In the following section, we analyze the sensitivity of the output $N$ of (10) with respect to the disturbances $X$ and $W$.

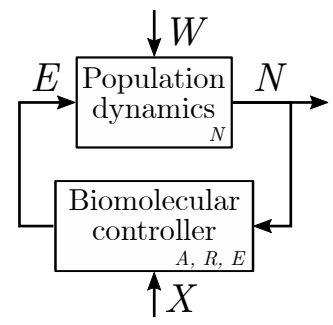

Fig. 2. Block diagram of nonlinear system (10) with disturbance inputs $W$ and $X$ and output $N$. The population dynamics block has state $N$, and the biomolecular controller block has states $A, R$, and E.

\section{Closed Loop Sensitivity Analysis}

We now analyze the sensitivity of the cell population density $N$ with respect to the environmental disturbance $W$ and the internal resource disturbance $X$. To achieve this, we linearize the nonlinear system (10) about an equilibrium point and compute the transfer functions from $W$ and $X$ to $N$. We then use these sensitivity transfer functions to analyze the trade-off between robustness to step disturbances in $W$ and in $X$.

\section{A. Linearization}

Let $\left(N_{0}, A_{0}, R_{0}, E_{0}\right)$ be an equilibrium point of system (10) in the positive orthant corresponding to constant nonnegative input levels $X_{0}$ and $W_{0}$. Let $\delta_{W}=$ $W-W_{0}$ and $\delta_{X}=X-X_{0}$ be small perturbations (disturbances) to the nominal inputs $X_{0}$ and $W_{0}$, and let $\delta_{N}=N-N_{0}, \delta_{A}=A-A_{0}, \delta_{R}=R-R_{0}$, and $\delta_{E}=E-E_{0}$ be the corresponding perturbations to the state of system (10). Then, the dynamics of these perturbations can be obtained by the linearization of system (10) about equilibrium point $\left(N_{0}, A_{0}, R_{0}, E_{0}\right)$ as

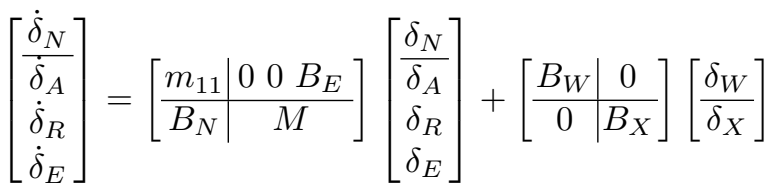

$$
\begin{aligned}
& y=\left[\begin{array}{llll}
1 & 0 & 0 & 0
\end{array}\right]\left[\begin{array}{l}
\frac{\delta_{N}}{\delta_{A}} \\
\delta_{R} \\
\delta_{E}
\end{array}\right]
\end{aligned}
$$

where $m_{11}=\mu\left(1-\frac{2 N_{0}+W_{0}}{K}\right)-\left(\gamma_{N}+G E_{0}\right)=$ $\frac{-\mu N_{0}}{K}$, which simplifies using the equilibrium point constraint in (10a). Additionally, $B_{E}=-G N_{0}, B_{N}=$ $\left[\frac{\alpha_{A} \frac{X_{0}}{K_{A}}}{1+\frac{X_{0}}{K_{A}}} \quad 0 \quad 0\right]^{T}$, and

$$
M=\left[\begin{array}{ccc}
-\gamma_{A}-\gamma \frac{R_{0}}{K_{R_{2}}} & -\gamma \frac{A_{0}}{K_{R_{2}}} & 0 \\
-\gamma \frac{R_{0}}{K_{R_{2}}} & -\gamma\left(1+\frac{A_{0}}{K_{R_{2}}}\right) & 0 \\
\alpha_{E}^{*} R_{0} X_{0} & \alpha_{E}^{*} A_{0} X_{0} & -\gamma
\end{array}\right],
$$

where $\alpha_{E}^{*}=\frac{\frac{\alpha_{E}}{K_{E} K_{2}}}{\left(1+\frac{A_{0} R_{0} X_{0}}{K_{E} K_{R_{2}}}\right)^{2}}$. The disturbance input matrices are given as $B_{W}=\frac{-\mu N_{0}}{K}$, and

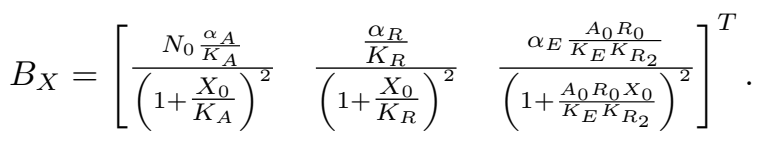

Next, we take advantage of the structure of (11) and rewrite the system as a feedback interconnection between of the growth dynamics $\delta_{N}$ and the biomolecular controller dynamics as in Figure 2. The population controller takes $\delta_{E}$ and $\delta_{W}$ as inputs and gives $\delta_{N}$ as the output, and the biomolecular controller takes $\delta_{N}$ and $\delta_{X}$ as inputs and gives $\delta_{E}$ as the output. Then, we have the 
state space representation of the population dynamics

$$
\begin{aligned}
\frac{d}{d t} \delta_{N} & =m_{11}\left[\delta_{N}\right]+\left[\begin{array}{ll}
B_{W} & B_{E}
\end{array}\right]\left[\begin{array}{l}
\delta_{W} \\
y_{C}
\end{array}\right] \\
y_{P} & =[1]\left[\delta_{N}\right],
\end{aligned}
$$

and the state space representation for the biomolecular controller as

$$
\begin{aligned}
\frac{d}{d t}\left[\begin{array}{l}
\delta_{A} \\
\delta_{R} \\
\delta_{E}
\end{array}\right] & =[M]\left[\begin{array}{l}
\delta_{A} \\
\delta_{R} \\
\delta_{E}
\end{array}\right]+\left[\begin{array}{ll}
B_{N} & B_{X}
\end{array}\right]\left[\begin{array}{l}
y_{P} \\
\delta_{X}
\end{array}\right] \\
y_{C} & =\left[\begin{array}{lll}
0 & 0 & 1
\end{array}\right]\left[\begin{array}{l}
\delta_{A} \\
\delta_{R} \\
\delta_{E}
\end{array}\right] .
\end{aligned}
$$

\section{B. Problem formulation}

We wish to analyze how the disturbances $\delta_{W}$ and $\delta_{X}$ affect the output $\delta_{N}$. In this system, it is possible to change the degradation rate $\gamma_{A}$ of $A$ by changing the $\mathrm{pH}$ of the media [26]. Additionally, we will show that $\frac{1}{\gamma_{A}}$ is related to the closed loop gain, and will examine how changing $\gamma_{A}$ affects the sensitivity of the output $\delta_{N}$ to disturbances $\delta_{W}$ and $\delta_{X}$. To this end, we compute transfer functions from $\delta_{W}$ and $\delta_{X}$ to $\delta_{N}$ using (12) and (13). Using (12), the transfer functions from $\delta_{E}$ to $\delta_{N}$ and from $\delta_{W}$ to $\delta_{N}$ are given by $P(s)=[1]\left(s-m_{11}\right)^{-1} B_{E}$ and $P_{W}(s)=[1]\left(s-m_{11}\right)^{-1} B_{W}$, respectively, using the standard formula. Then, simplifying, we have

$$
\delta_{N}(s)=\underbrace{\frac{-G N_{0}}{s+\mu \frac{N_{0}}{K}}}_{P(s)} \delta_{E}(s)+\underbrace{\frac{-\mu \frac{N_{0}}{K}}{s+\mu \frac{N_{0}}{K}}}_{P_{W}(s)} \delta_{W}(s) .
$$

Additionally, the transfer function of the biomolecular controller from $\delta_{N}$ to $\delta_{E}$ using (13) is $\frac{\delta_{E}(s)}{\delta_{N}(s)}=$ $[0,0,1](s I-M)^{-1} B_{N}$, which simplifies to become

$$
C(s)=\frac{\delta_{E}(s)}{\delta_{N}(s)}=\frac{\alpha^{*} R_{0} X_{0}^{2}}{s^{2}+s \xi+\gamma\left(\gamma_{A}+\frac{\gamma_{A} A_{0}+\gamma R_{0}}{K_{R_{2}}}\right)}
$$

where $\alpha^{*}=\frac{\alpha_{A} \alpha_{E} \frac{R_{0} X_{0}^{2}}{K_{A} K_{E} K_{R_{2}}}}{\left(1+\frac{X_{0}}{K_{A}}\right)\left(1+\frac{A_{0} R_{0} X_{0}}{K_{E} K_{R_{2}}}\right)^{2}}$ and $\xi=$ $\left(\gamma \frac{A_{0}+R_{0}}{K_{R_{2}}}+\gamma+\gamma_{A}\right)$. The transfer function from the disturbance $\delta_{X}$ to $\delta_{E}$ is given as $C_{X}(s)=\frac{\delta_{E}(s)}{\delta_{X}(s)}=$ $[0,0,1](s I-M)^{-1} B_{X}$. To simplify and for ease of analysis later, we pull $C_{X}(s)$ through $C(s)$ and define $K_{C}(s)=\frac{C_{X}(s)}{C(s)}$, which is shown in Figure 3 and is given by

$$
\begin{aligned}
& K_{C}(s)=\frac{A_{0}\left(\frac{K_{A}}{X_{0}}+1\right)}{X_{0} \alpha_{A}}\left(s+\gamma_{A}+\gamma \frac{R_{0}}{K_{R_{2}}}\right)+\frac{\frac{N_{0}}{X_{0}}}{1+\frac{X_{0}}{K_{A}}} \\
& +\left(\frac{s+\gamma_{A}}{s+\gamma}\right) \frac{A_{0}\left(1+\frac{K_{A}}{X_{0}}\right)}{\alpha_{A}}\left(\frac{\gamma A_{0}}{X_{0} K_{R_{2}}}+\frac{\frac{\alpha_{R}}{K_{R} R_{0}}}{\left(1+\frac{X_{0}}{K_{R}}\right)^{2}}\right)
\end{aligned}
$$

In the closed loop system, we wish to evaluate how tuning the parameter $\gamma_{A}$ affects the performance of the biomolecular controller. To this end, we calculate the loop gain of the closed loop system $L(s)=P(s) C(s)$, which is given as

$$
L(s)=\frac{-G N_{0} \alpha^{*} R_{0} X_{0}^{2}}{\left(s+\mu \frac{N_{0}}{K}\right)\left(s^{2}+s \xi+\gamma\left(\gamma_{A}+\frac{\gamma_{A} A_{0}+\gamma R_{0}}{K_{R_{2}}}\right)\right)} .
$$

Thus, the loop gain $L(s)$ may be tuned by varying $\gamma_{A}$.

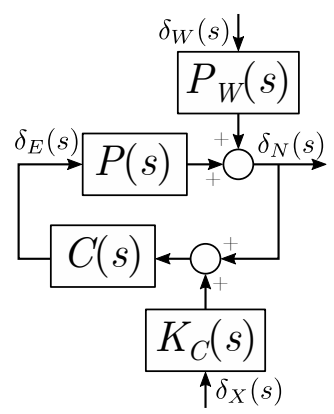

Fig. 3. Block diagram of the closed loop system rearranged such that the perturbation $\delta_{X}(s)$ appears on the feedback path. This form is helpful for analyzing the closed loop sensitivity of $\delta_{N}(s)$ to perturbations in the combination of $\delta_{W}(s)$ and $\delta_{X}(s)$.

Next, we analyze a weighted sensitivity function that is a linear combination of the sensitivity of $\delta_{N}$ to $\delta_{X}$ and to $\delta_{W}$ and show in Theorem 1 that this function has a strictly positive lower bound.

Definition 1. Let the magnitude of the sensitivity of the output $\delta_{N}$ to the disturbance $\delta_{W}$ be

$$
S(s)=\left|\frac{\delta_{N}(s)}{\delta_{W}(s)}\right|
$$

and the normalized magnitude of the sensitivity of the output $\delta_{N}$ to the disturbance $\delta_{X}$ be

$$
T(s)=\left|\left(\frac{X_{0}}{N_{0}}\right) \frac{\delta_{N}(s)}{\delta_{X}(s)}\right| .
$$

The total weighted sensitivity function is defined as

$$
\zeta(s)=S(s)+T(s) .
$$

The value of $\zeta(s)$ determines the magnitude of the response of $\delta_{N}$ with respect to the combination of the disturbances $\delta_{W}$ and $\delta_{X}$.

Theorem 1. Consider system (11) where $\delta_{W}$ and $\delta_{X}$ are step disturbances, then the weighted sensitivity function $\zeta$ evaluated at $s=0$ has the lower bound

$$
\zeta(0) \geq 1 \text {. }
$$

Proof. First we evaluate $S(s)$ at $s=0$. From the block diagram in Figure 3, $S(s)=\left|\frac{N(s)}{W(s)}\right|=\left|\frac{P_{W}(s)}{1-L(s)}\right|$. At $s=0, P_{W}(0)=-1$, so $S(0)=\frac{1}{|1-L(0)|}$. Next, we 
evaluate $T(s)$ at $s=0$. From the block diagram in Figure 3, $T(0)=\left|\frac{X_{0}}{N_{0}} \frac{\delta_{N}(s)}{\delta_{X}(s)}\right|=\left|\frac{\frac{X_{0}}{N_{0}} K_{C}(0) L(0)}{1-L(0)}\right|$ where $K_{C}(s)$ is given in (16). To find a bound for $T(0)$, we note that $A_{0}=N_{0} \frac{\alpha_{A}}{\gamma_{A}+\gamma \frac{R_{0}}{K_{R_{2}}}}\left(\frac{X_{0}}{K_{A}+X_{0}}\right)$ from (10b). Then, substituting and evaluating (16) at $s=0$ and simplifying, $K_{C}(0)$ is given as

$$
\begin{array}{r}
K_{C}(0)=\frac{N_{0}}{X_{0}}\left(1+\frac{1}{1+\frac{X_{0}}{K_{A}}}\right)+\frac{N_{0} \gamma_{A}}{\left(\gamma_{A}+\gamma \frac{R_{0}}{K_{R_{2}}}\right)} \\
\cdot\left(\frac{\frac{N_{0} \alpha_{A}}{X_{0}+K_{A}}}{\gamma_{A} K_{R_{2}}+\gamma R_{0}}+\frac{\frac{\alpha_{R}}{\gamma K_{R} R_{0}}}{\left(1+\frac{X_{0}}{K_{R}}\right)^{2}}\right) \geq \frac{N_{0}}{X_{0}}
\end{array}
$$

since all concentrations are nonnegative. Substituting the relations for $T(0), S(0)$, and the inequality (17) into the definition of $\zeta$ and simplifying, we have

$$
\zeta(0)=\frac{1+\frac{X_{0}}{N_{0}} K_{C}(0)|L(0)|}{|1-L(0)|} \geq \frac{1+|L(0)|}{|1-L(0)|}=1
$$

since $L(0) \leq 0$.

Theorem 1 shows that for any stable equilibrium point of (10), it is impossible to tune the population controller to reject step disturbances from both the environment $\delta_{W}$ and resource fluctuations $\delta_{X}$, i.e. $S(0)$ and $T(0)$ cannot both be made small simultaneously. In Figure 4, $S(0), T(0)$, and $\zeta(0)$ are shown for different values of the parameter $\gamma_{A}$, and $\zeta(0)$ is bounded from below by $0 \mathrm{~dB}$. Future work may consider the system for $s \neq 0$ and compare the sensitivities at each value of $s$.

\section{Remarks on Stability of the Closed Loop System}

The calculation of sensitivity functions in Section III only have meaning when the closed loop system is stable, since the output after a perturbation must remain within a neighborhood of the equilibrium point. Here we find conditions where the closed loop is guaranteed to be stable using the closed loop linearized transfer function. From (14) and (15), the closed loop characteristic polynomial is given as

$$
\begin{aligned}
s^{3}+s^{2}\left(\frac{\mu N_{0}}{K}+\xi\right)+s(b+ & \left.\xi \frac{\mu N_{0}}{K}\right) \\
& +\frac{\mu N_{0}}{K} b-\alpha^{*} G N_{0},
\end{aligned}
$$

where $b=\gamma\left(\gamma_{A}+\frac{\gamma_{A} A_{0}+\gamma R_{0}}{K_{R_{2}}}\right)$. From the RouthHurwitz criterion for a 3 rd degree polynomial, the closed

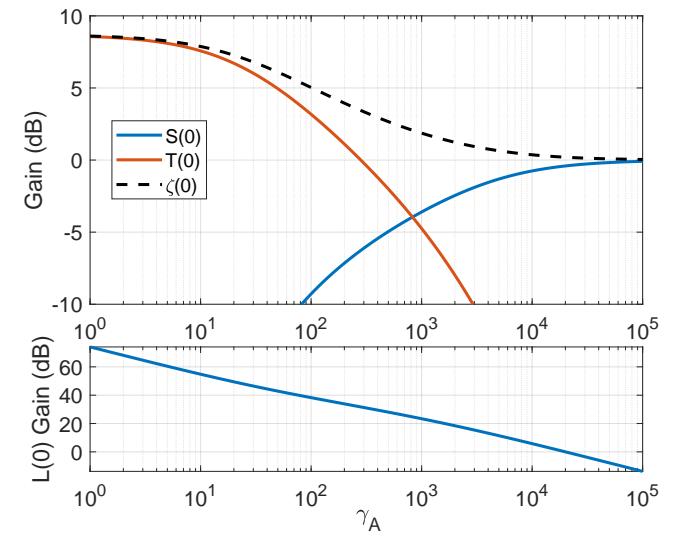

Fig. 4. Values of the sensitivity functions $S, T$, and $\zeta$ about the equilibrium point of (10) evaluated at $s=0$ for different values of $\gamma_{A}$. For these parameters, the magnitude of the loop gain $|L(0)|$ decreases as $\gamma_{A}$ is increased. $\zeta(0)$ shows the lower bound of $0 \mathrm{~dB}$ derived in Theorem 1. Parameters used for the simulations are $\mu=4.1 \mathrm{~h}^{-1}$, $K=2000, G=1 \mathrm{~h}^{-1} \mathrm{nM}^{-1}, \gamma_{N}=0.1 \mathrm{~h}^{-1}, \gamma=1 \mathrm{~h}^{-1}, k_{A}=$ $2 \mathrm{~h}^{-1}, k_{R}=1 \mathrm{~h}^{-1}, k_{E}=1 \mathrm{~h}^{-1}, D_{A_{t o t}}=100 \mathrm{nM}, D_{R_{t o t}}=$ $10 \mathrm{nM}, D_{E_{t o t}}=10 \mathrm{nM}, K_{A}=15 \mathrm{nM}, K_{R}=20 \mathrm{nM}, K_{E}=$ $300 \mathrm{nM}^{2}, K_{R_{2}}=1000 \mathrm{nM}, X_{0}=55 \mathrm{nM}$. The equilibrium point of the system for all values of $\gamma_{A}$ shown exhibit asymptotic stability.

loop system is stable if and only if

$$
\begin{aligned}
& \frac{\mu N_{0}}{K}+\gamma_{A}+\gamma\left(1+\frac{A_{0}+R_{0}}{K_{R_{2}}}\right)>0 \\
& \gamma \frac{\mu}{K}\left(\gamma_{A}+\frac{\gamma_{A} A_{0}+\gamma R_{0}}{K_{R_{2}}}\right)>\alpha^{*} G \\
& \gamma\left(\frac{\mu N_{0}}{K}+\gamma\left(1+\frac{A_{0}+R_{0}}{K_{R_{2}}}\right)+\gamma_{A}\right) \\
& \quad \cdot\left(\gamma \gamma_{A}+\gamma \frac{\gamma_{A} A_{0}+\gamma R_{0}}{K_{R_{2}}}+\frac{\mu N_{0}}{K}\left(\gamma+\gamma_{A}\right)\right)>\alpha^{*} G N_{0} .
\end{aligned}
$$

If we also assume that $\frac{A_{0}+R_{0}}{K_{R_{2}}} \ll 1$ and $\frac{\gamma R_{0}}{\gamma_{A} K_{R_{2}}} \ll 1$, as before, then (18) simplifies to

$$
\begin{aligned}
& \frac{\mu N_{0}}{K}+\gamma_{A}+\gamma>0 \\
& \gamma_{A} \gamma \frac{\mu}{K}>\alpha^{*} G \\
& \gamma\left(\frac{\mu N_{0}}{K}+\gamma_{A}+\gamma\right)\left(\gamma_{A}+\frac{\mu N_{0}\left(\gamma+\gamma_{A}\right)}{K}\right)>\alpha^{*} G N_{0} .
\end{aligned}
$$

Since $\alpha^{*} \leq \frac{\alpha_{A} \alpha_{E}}{A_{0}}$ and $N_{0} \leq K$, if $\gamma_{A}>\frac{\alpha_{A} \alpha_{E} G K}{\mu \gamma A_{0}}$ and $\gamma_{A}>\sqrt{\frac{\alpha_{A} \alpha_{E} G K}{A_{0} \gamma}}$, then all inequalities in (19) are satisfied. Since $A_{0}$ is bounded away from 0 , then the closed loop system is stable for large enough $\gamma_{A}$, i.e. when the feedback gain is sufficiently small.

\section{DISCUSSION}

We derived a mechanistic model for a cellular population controller, which is the most common population 
bioRxiv preprint doi: https://doi.org/10.1101/2020.06.04.134932; this version posted June 5, 2020. The copyright holder for this preprint (which was not certified by peer review) is the author/funder, who has granted bioRxiv a license to display the preprint in perpetuity. It is made available under aCC-BY-ND 4.0 International license.

control system in bacteria [25], [31]. We showed that, in this controller architecture, there is a trade-off between robustness to environmental disturbances and robustness to perturbations in available resources to the genetic circuit. Placing different genetic circuit components in multiple cells has been proposed as a possible solution to relieve some resource sharing effects for larger genetic circuits [15], [21], [32]. However, our analysis shows that when fluctuations in cellular resources are considered, it is not possible to tune the population controller to be both robust to extracellular disturbances and to intracellular disturbances. Thus, if distributed biomolecular systems are to be used to mitigate resource loading within a single cell in order to make larger genetic circuits, the population controller should be designed to ensure an acceptable level of robustness to environmental disturbances and to perturbations due to resource fluctuations.

\section{ACKNOWLEDGMENT}

The authors thank Ted Grunberg for helpful discussions.

\section{REFERENCES}

[1] C. T. Y. Chan, J. W. Lee, D. E. Cameron, C. J. Bashor, and J. J. Collins, "Deadman' and 'Passcode' microbial kill switches for bacterial containment," Nat Chem Biol, vol. 12, no. 2, pp. 82-86, Feb. 2016.

[2] Y. Qian, C. McBride, and D. D. Vecchio, "Programming Cells to Work for Us," Annual Review of Control, Robotics, and Autonomous Systems, vol. 1, no. 1, p. null, 2018.

[3] P. E. M. Purnick and R. Weiss, "The second wave of synthetic biology: from modules to systems," Nat Rev Mol Cell Biol, vol. 10, no. 6, pp. 410-422, June 2009.

[4] O. Wright, G.-B. Stan, and T. Ellis, "Building-in biosafety for synthetic biology," Microbiology, vol. 159, no. 7, pp. 1221-1235, 2013.

[5] A. Gyorgy, J. Jiménez, J. Yazbek, H.-H. Huang, H. Chung, R. Weiss, and D. Del Vecchio, "Isocost Lines Describe the Cellular Economy of Genetic Circuits," Biophysical Journal, vol. 109, no. 3, pp. 639-646, Aug. 2015.

[6] Y. Qian, H.-H. Huang, J. I. Jiménez, and D. Del Vecchio, "Resource Competition Shapes the Response of Genetic Circuits," ACS Synth. Biol., vol. 6, no. 7, pp. 1263-1272, July 2017.

[7] O. Borkowski, F. Ceroni, G.-B. Stan, and T. Ellis, "Overloaded and stressed: whole-cell considerations for bacterial synthetic biology," Current Opinion in Microbiology, vol. 33, pp. 123130, Oct. 2016.

[8] S. Klumpp and T. Hwa, "Bacterial growth: global effects on gene expression, growth feedback and proteome partition," Curr. Opin. Biotechnol., vol. 28, pp. 96-102, Aug. 2014.

[9] F. Ceroni, R. Algar, G.-B. Stan, and T. Ellis, "Quantifying cellular capacity identifies gene expression designs with reduced burden," Nature Methods, vol. 12, no. 5, p. 415, Apr. 2015.

[10] H.-H. Huang, Y. Qian, and D. D. Vecchio, "A quasi-integral controller for adaptation of genetic modules to variable ribosome demand," bioRxiv, p. 336271, June 2018.

[11] T. Shopera, L. He, T. Oyetunde, Y. J. Tang, and T. S. Moon, "Decoupling Resource-Coupled Gene Expression in Living Cells," ACS Synth. Biol., vol. 6, no. 8, pp. 1596-1604, Aug. 2017.

[12] A. P. S. Darlington, J. Kim, J. I. Jiménez, and D. G. Bates, "Dynamic allocation of orthogonal ribosomes facilitates uncoupling of co-expressed genes," Nature Communications, vol. 9, no. 1, p. 695 , Feb. 2018.
[13] J. Macia, R. Manzoni, N. Conde, A. Urrios, E. d. Nadal, R. Solé, and F. Posas, "Implementation of Complex Biological Logic Circuits Using Spatially Distributed Multicellular Consortia," PLOS Computational Biology, vol. 12, no. 2, p. e1004685, Feb. 2016.

[14] F. Annunziata, A. Matyjaszkiewicz, G. Fiore, C. S. Grierson, L. Marucci, M. di Bernardo, and N. J. Savery, "An Orthogonal Multi-input Integration System to Control Gene Expression in Escherichia coli," ACS Synth. Biol., vol. 6, no. 10, pp. 18161824, Oct. 2017.

[15] R. Tsoi, F. Wu, C. Zhang, S. Bewick, D. Karig, and L. You, "Metabolic division of labor in microbial systems," PNAS, vol. 115, no. 10, pp. 2526-2531, Mar. 2018.

[16] S. Basu, Y. Gerchman, C. H. Collins, F. H. Arnold, and R. Weiss, "A synthetic multicellular system for programmed pattern formation," Nature, vol. 434, no. 7037, p. 1130, Apr. 2005.

[17] X. Cao, J. J. Hamilton, and O. S. Venturelli, "Understanding and Engineering Distributed Biochemical Pathways in Microbial Communities," Biochemistry, vol. 58, no. 2, pp. 94-107, Jan. 2019.

[18] O. Kanakov, R. Kotelnikov, A. Alsaedi, L. Tsimring, R. Huerta, A. Zaikin, and M. Ivanchenko, "Multi-Input Distributed Classifiers for Synthetic Genetic Circuits," PLOS ONE, vol. 10, no. 5, p. e0125144, May 2015.

[19] X. Ren, A. Baetica, A. Swaminathan, and R. M. Murray, "Population regulation in microbial consortia using dual feedback control," in 2017 IEEE 56th Annual Conference on Decision and Control (CDC), Dec. 2017, pp. 5341-5347.

[20] S. R. Scott, M. O. Din, P. Bittihn, L. Xiong, L. S. Tsimring, and J. Hasty, "A stabilized microbial ecosystem of self-limiting bacteria using synthetic quorum-regulated lysis," Nature Microbiology, vol. 2, no. 8, p. 17083, Aug. 2017.

[21] M. Thommes, T. Wang, Q. Zhao, I. C. Paschalidis, and D. Segrè, "Designing Metabolic Division of Labor in Microbial Communities," mSystems, vol. 4, no. 2, pp. e00 263-18, Apr. 2019.

[22] A. Urrios, J. Macia, R. Manzoni, N. Conde, A. Bonforti, E. de Nadal, F. Posas, and R. Solé, "A Synthetic Multicellular Memory Device," ACS Synth. Biol., vol. 5, no. 8, pp. 862-873, Aug. 2016.

[23] C. McBride and D. Del Vecchio, "Resource Sensor Design for Quantifying Resource Competition in Genetic Circuits," in Proceedings of IEEE Conference on Decision and Control (CDC) 2018, Miami Beach, FL, USA, Dec. 2018.

[24] A. L. Schaefer, C. R. Lappala, R. P. Morlen, D. A. Pelletier, T.Y. S. Lu, P. K. Lankford, C. S. Harwood, and E. P. Greenberg, "LuxR- and LuxI-Type Quorum-Sensing Circuits Are Prevalent in Members of the Populus deltoides Microbiome," Appl Environ Microbiol, vol. 79, no. 18, pp. 5745-5752, Sept. 2013.

[25] W.-L. Ng and B. L. Bassler, "Bacterial Quorum-Sensing Network Architectures," Annu Rev Genet, vol. 43, pp. 197-222, 2009.

[26] L. You, R. S. Cox, R. Weiss, and F. H. Arnold, "Programmed population control by cell-cell communication and regulated killing," Nature, vol. 428, no. 6985, p. 868, Apr. 2004.

[27] M. H. Zwietering, I. Jongenburger, F. M. Rombouts, and K. v. t. Riet, "Modeling of the Bacterial Growth Curve," Appl. Environ. Microbiol., vol. 56, no. 6, pp. 1875-1881, June 1990.

[28] D. Del Vecchio and R. M. Murray, Biomolecular Feedback Systems. Princeton: Princeton University Press, Oct. 2014.

[29] P. Érdi and J. Tóth, Mathematical Models of Chemical Reactions: Theory and Applications of Deterministic and Stochastic Models. Manchester University Press, 1989, google-Books-ID: iDu8AAAAIAAJ.

[30] H. K. Khalil, Nonlinear Systems, 3rd ed. Upper Saddle River, N.J: Pearson, Dec. 2001.

[31] R. Tsoi, Z. Dai, and L. You, "Emerging strategies for engineering microbial communities," Biotechnology Advances, vol. 37, no. 6, p. 107372, Nov. 2019.

[32] Y. Xiang, N. Dalchau, and B. Wang, "Scaling up genetic circuit design for cellular computing: advances and prospects," Nat Comput, vol. 17, no. 4, pp. 833-853, 2018. 